\title{
ASYMMETRY OF DOMINANT AND NON-DOMINANT SHOULDERS IN UNIVERSITY LEVEL MEN AND WOMEN VOLLEYBALL PLAYERS
}

rewiev paper

(1) University School of Physical Education in Wroclaw

DOI: https://doi.org/10.5114/hm.2019.85095

\section{OLGA ZUZGINA, MAXIMILIAN M. WDOWSKI}

Faculty of Health and Life Sciences, Coventry University, Coventry, UK

\begin{abstract}
Purpose. Volleyball involves repetitive forceful actions predominantly of one arm, which could eventually lead to an injury or shoulder pathology. The aim of the study was to compare the range of motion and strength in the dominant and non-dominant shoulders of university level volleyball players, with the objective of examining any differences between sexes.

Methods. A total of 19 university level volleyball players (9 men: $81.3 \pm 8.0 \mathrm{~kg}, 21 \pm 1$ years; 10 women: $66.0 \pm 8.2 \mathrm{~kg}$, $19 \pm 1$ years) participated. The passive internal and external range of motion of the dominant and non-dominant spiking shoulders were measured with a goniometer. The shoulder strength tests were performed on an isokinetic dynamometer at the speed of $60 \% \mathrm{~s}$ in the concentric mode of contraction. Shoulder internal and external rotator concentric strength, range of motion, and internal/external rotation strength ratios were tested between arm dominance and sex with the use of 2-way mixed design ANOVA.
\end{abstract}

Results. Internal and external rotation strength was asymmetrical in both men and women, in favour of the dominant side $(p<0.05)$. Both men and women displayed asymmetrical external/internal ratios $(p<0.05)$, with greater ratios present in the non-dominant side.

Conclusions. University level men and women volleyball players exhibit similar levels of significant asymmetry in the internal/external rotation strength between dominant and non-dominant spiking arms. Furthermore, subject-specific responses in strength asymmetries suggest further investigation at the individual level. Isokinetic shoulder screening could be used to reveal information about possible risk factors for shoulder injuries.

Key words: injury, biomechanics, dynamometer, sport

\section{Introduction}

Inter-limb asymmetry compares the movement of one limb with respect to the other [1]. Research indicates that inter-limb differences measured across a range of tasks exert a detrimental effect on physical and sport performance [2], and have potentially injurious consequences [3]. In demanding overhead sports like tennis, basketball, or baseball, the asymmetrical nature of the throwing or serving movements can result in up to $44 \%$ of injury cases being classified as shoulder injuries [4]. Furthermore, Seminati and Minetti [5] argued that common shoulder injuries in overhead sports (impingement syndrome, suprascapular neuropathy, shoulder instability, and shoulder muscle strains) were caused by repetitive overhead movements.
As a consequence of the interest in injury risk and occurrence, asymmetries of $<10 \%$ have been proposed as the target for when athletes are returning to or currently competing in sport $[2,6,7]$, and both athlete and non-athlete populations who exhibit inter-limb asymmetries greater than $15 \%$ have been associated with increased injury incidence and reduced performance when compared with groups who score below this threshold [8-11]. However, given the subject-specific and experimental test-specific nature of asymmetries [12], caution should be used when applying these thresholds across different experimental tests and cohorts.

Volleyball is an example of an overhead sport where acute and overuse injuries might occur as a result of the sport-specific movements such as spiking, serving,

Correspondence address: Maximilian M. Wdowski, Level 2, Alison Gingell Building, Faculty of Health and Life Sciences, Coventry University, Coventry, CV1 5FB, United Kingdom, e-mail: ac6071@coventry.ac.uk

Received: June 26, 2018

Accepted for publication: April 25, 2019

Citation: Zuzgina O, Wdowski MM. Asymmetry of dominant and non-dominant shoulders in university level men and women volleyball players. Hum Mov. 2019;20(4):19-27; doi: https://doi.org/10.5114/hm.2019.85095. 
and blocking [13]. A 4-year data collection from the International Volleyball Federation (FIVB, Fédération Internationale de Volleyball), where the medical staff of the participating teams were required to report all newly incurred injuries during all major FIVB tournaments (World Championships, World Cup, World Grand Prix, World League, Olympic Games), identified the shoulder as one of the most common overuse injury sites that occur in volleyball athletes, behind the knee [13]. Considering the asymmetric nature of shoulder movements in volleyball, one would expect that volleyball practice and match play can cause strength imbalances that present in the form of strength asymmetries between the dominant (D) and non-dominant (ND) shoulders. Isokinetic strength testing can play an essential part in the comprehensive evaluation and rehabilitation of a patient with a shoulder injury [14]. Evaluating the strength of shoulder external rotators (ER) and internal rotators (IR) has been suggested to be most informative because those muscle groups are responsible for dynamic stabilisation of the glenohumeral joint [15]. One possible mechanism leading to shoulder injury may be a strength imbalance between the IR and ER muscles [16], which can be assessed by using isokinetic strength testing [17]. Strength imbalances have been measured as ratios between intra-limb muscles [16, 17]. Differences in IR and ER strength ratios appear to be related to injury in sports that involve overhead throwing activities, such as handball [18] and volleyball [19]. Therefore, gaining an understanding of potential intra-limb strength ratios in shoulder function and strength through isokinetic measurements is imperative for overhead sports.

To further highlight the importance of understanding asymmetries in shoulder strength, Reinold and Gill [20], and later other studies [21-23] stated that an overhead athlete is at greater risk of having an injury if their D shoulder is more than 9\% stronger in the IR and more than $14 \%$ weaker in ER than the ND shoulder. Hadzic et al. [17] highlighted that studies had previously examined ER and IR strength asymmetries between D and ND in volleyball players. They stated that these studies were generally focused on elite level volleyball playing men and that the findings were somewhat inconsistent. For example, some studies [21, 22] did not report strength asymmetry and others [23-25] concluded that IR strength on the D side was greater than that on the ND side. Discrepancy also exists with regard to ER strength, as greater values have been reported on either the ND side [23, 25] or the D side [26]. Although research has provided evidence of positionspecific injury risks, such as outside players having increased risk of shoulder injury over all other playing positions [13], current research has not associated playing position with an abnormal shoulder strength ratio or strength asymmetry [17]. There is also limited information on sex differences; a study by Hadzic et al. [17] on high level volleyball players speculated that women volleyball players could have a lower risk of developing shoulder-related problems than men. However, they only found the sex difference in women without a previous shoulder injury, potentially suggesting that the observation had limited implications to shoulder injury post recovery.

The constant repetitive technical movements in volleyball may result in functional, morphological, and biomechanical alterations of the D shoulder (preferred arm during a volleyball serve and spike), such as changes in the range of motion (ROM), muscle strength, the shoulder capsule and the scapula [27, 28]. Overhead sport athletes who perform a throwing motion similar to the volleyball spike have been shown to demonstrate increases in the ROM of ER and reductions in the ROM of IR in their D shoulders compared with the opposite side [16, 29]. However, definitive conclusions about these patterns and their associations with injury have not been established in volleyball [3].

As a consequence of the inconsistent and limited findings regarding sex differences [17], IR/ER ratios [21], and D/ND strength asymmetries [21-25], as well as a narrow breadth of populations examined [17], there is a requirement for further studies to confirm the presence of asymmetries and sex differences at skill level outside of elite and high level volleyball players. One specific population are university/college level volleyball players. Previous research has highlighted an increased injury prevalence at university/college owing to the increase in intensity and level of practice when compared with non-athletes [30,31]. Furthermore, the associated injuries sustained during university level sport may limit future physical activity levels [31].

Therefore, the aim of the study was to investigate the presence of asymmetries in the ROM and strength in the D and ND shoulders of university level men and women volleyball players. We hypothesised that men and women would display similar levels of significant asymmetries in strength and ROM between D and ND shoulders. The findings in this study may contribute to understanding the quantity of asymmetries present for screening purposes and potential injury risk. 


\section{Material and methods}

\section{Participants}

Overall, 19 university level volleyball players (9 men: $81.3 \pm 8.0 \mathrm{~kg}, 21 \pm 1$ years; 10 women: $66.0 \pm 8.2 \mathrm{~kg}$, $19 \pm 1$ years) participated in the study. The subjects trained $6.2 \pm 2.1$ hours a week (generally 2 skill-based sessions and 1 strength and conditioning session) and had played volleyball on a regular basis for $7.3 \pm 3.2$ years. The number of matches played each week or time on court per match were not collected. Participants were excluded if they were currently injured, not currently performing, had a previous history of shoulder surgery, had shoulder pain, instability, impingement pain, or had visited a clinician regarding shoulder problems in the previous 12 months. Each participant completed a questionnaire concerning the sex, hand dominance, years of practicing volleyball, the playing position, hours of training, and incidence of previous shoulder injuries. The $\mathrm{D}$ arm was defined as the $\mathrm{D}$ hand used to spike and serve.

\section{Procedures}

In an attempt to reduce the effect of match play or training fatigue, no volleyball practice or upper body training were allowed 24 hours before the testing. All testing was performed in the daytime hours of 10:00-14:00 in an indoor university laboratory space at a temperature of approximately $18-21^{\circ} \mathrm{C}$. Prior to testing, the participant performed a 15-minute selfdirected warm-up, usually a combination of heart raising and dynamic volleyball specific movements. The passive ROM of IR and ER was measured by an experienced (3 years of clinical experience) sports therapist using a standard baseline goniometer (Fabrication Enterprises Inc., New York, USA). Participants were asked to lie supine with shoulder abducted to $90^{\circ}$, elbow flexed to $90^{\circ}$, and the wrist in neutral. One sports therapist was stabilising the player's scapula on the plinth to decrease the scapular elevation, which could affect the measurements. A second sports therapist passively moved the participant's D arm to rotate their arm back into ER to the end of their available range without discomfort and up to the point of where movement was resisted. The sports therapist then passively moved the participant's D arm to rotate their arm forward into IR to the end of their available range without discomfort and up to the point of where movement was resisted. The purpose of the passive trial repetition was to familiarize the participant with the requested motion. Following the passive trial, each subject performed one ER and one IR ROM trial with 1-minute rest between the movements.

As conducted by Kolber and Hanney [32], for the ER ROM trials, the participants were requested to rotate their arm back into ER to the end of their available range without discomfort. Once maximum range was achieved, the subject was asked to maintain the position while the angle was recorded with the goniometer. The second sports therapist measured the athlete's shoulder ER by positioning the axis of goniometer over the elbow olecranon process, with the stationary arm aligned vertically and the moving arm aligned along to ulna styloid process. Once the measurement was recorded, the participants moved their shoulder to a neutral position.

For the IR ROM trials, the subjects were requested to rotate their arm forward into IR to the end of their available range without discomfort. Once maximum range was achieved, the participant was asked to maintain the position while the angle was recorded with the goniometer. The second sports therapist measured the participant's shoulder IR by positioning the axis of goniometer over the elbow olecranon process, with the stationary arm aligned vertically and the moving arm aligned along to ulna styloid process. The same process of the sports therapist passively moving the arm followed by the measurement trials for IR and ER was then repeated in the ND arm.

The shoulder strength testing was performed by the same one researcher for each participant on an isokinetic strength dynamometer (Kin-Com AP Muscle Testing Systems, Chattanooga Group, Tennessee, USA). Before measuring the strength of IR and ER, the gravity compensation was calibrated with the use of spirit level measurer (Torpedo level 11560, Screwfix, Yeovil, UK). The standard attachments were used to stabilise the participant's torso, shoulder, and elbow. The dynamometer system was set up before each test session to fit the allowable limits for the right and left arm. The internal and external rotation of $\mathrm{D}$ and ND shoulders were assessed in sitting position, with the arm abducted to $90^{\circ}$ and with the elbow flexed to $90^{\circ}$. The ND limb was measured first. The starting position was set to $60^{\circ}$ of external rotation, at the speed of $60 \% \mathrm{~s}$ in the concentric mode of contraction [17]. The familiarisation process was completed at $60 \%$ and by three sub-maximal trials in the selected speed, followed by 1-minute rest before the actual test. The decision to test by using one speed was made on the findings from previous studies, where differences in isokinetic variables were usually found 
at the lower velocity [3,23]. The internal rotation was tested first for the three repetitions, the external rotation was measured next. After a 5-minute break, which allowed to change the sides and calibrate the system, the testing was repeated on the D shoulder. The participant could see the torque curve on the screen during the test.

\section{Statistical analysis}

The highest peak torque value $(\mathrm{Nm})$ from three repetitions was used in the further data analysis. In order to compare the data with previous studies [17], relative measures of strength of the peak torque value were normalized for body weight $(\mathrm{Nm} / \mathrm{kg}$ ) (Equation 1). The concentric ER/IR strength ratio for $D$ and ND shoulder was calculated (Equation 2) along with the absolute and relative strength percent deficit (\%) for asymmetry in IR and ER (Equation 3).

$$
\text { relative torque }=\frac{\text { absolute torque }(\mathrm{Nm})}{\text { body weight }(\mathrm{kg})}
$$

$\mathrm{ER} / \mathrm{IR}$ strength ratio $=\frac{\text { external rotation peak torque }}{\text { internal rotation peak torque }}$

$\%$ deficit $=\frac{\text { higher peak torque }- \text { lower peak torque }}{\text { higher peak torque }} \times 100$

Normality was assessed for all variables with the Shapiro-Wilk test, with the data conforming to normal distribution. Separate 2-way mixed design ANOVA (with independent measures on sex and repeated measures on shoulder dominance) with a Bonferroni correction was used to assess differences between $\mathrm{ROM}$, relative and absolute IR and ER concentric strength, and relative and absolute ER/IR strength ratios. Independent measures $t$-tests were performed for relative and absolute strength deficit to test between sexes. All calculations were performed in SPSS software for Windows (version 24, IBM SPSS Statistics, Chicago, USA). The significance level of 0.05 was applied for all tests and partial eta-squared $\left(\eta p^{2}\right)$ effect sizes were calculated, with $0.001,0.06$, and 0.14 classified as small, medium, and large effect sizes, respectively [33].

\section{Ethical approval}

The research related to human use has complied with all the relevant national regulations and institutional policies, has followed the tenets of the Declaration of Helsinki, and has been approved by the Ethical Department of the Faculty of Health and Life Sciences, Coventry University.

\section{Informed consent}

Informed consent has been obtained from all individuals included in this study.

\section{Results}

ROM for both IR and ER was found to be similar between D and ND shoulders (ER: 0\%, $p=0.93, \eta p^{2}$ $=0.001$, small effect; IR: $5 \%, p=0.43, \eta p^{2}=0.078$, small effect), and between men and women (ER: $4 \%$, $p=0.20, \eta p^{2}=0.200$, large effect; IR: $2 \%, p=0.79$, $\eta p^{2}=0.010$, small effect) (Figure 1). For the results presented in Table 1, IR and ER absolute peak concentric torque was found to be significantly different between $D$ and ND shoulders (IR: $19 \%, p=0.001, \eta p^{2}=0.763$, large effect; ER: $7 \%, p=0.014, \eta p^{2}=0.548$, large effect), and between men and women (IR: $42 \%, p=0.002$, $\eta p^{2}=0.732$, large effect; ER: $49 \%, p=0.006, \eta p^{2}=$ 0.637 , large effect). Relative IR peak torque was observed to be significantly different between $\mathrm{D}$ and ND shoulders $\left(20 \%, p=0.001, \eta p^{2}=0.771\right.$, large effect), and between males and females $\left(29 \%, p=0.026, \eta p^{2}=\right.$ 0.480 , large effect). Relative ER peak concentric torque was observed to be significantly different between men and women $\left(35 \%, p=0.033, \eta p^{2}=0.454\right.$, large effect). Relative and absolute ER/IR ratios were significantly different between $\mathrm{D}$ and ND shoulders (relative: $23 \%, p=0.024, \eta p^{2}=0.489$, large effect; absolute: $17 \%$, $p=0.012, \eta p^{2}=0.570$, large effect). Relative and absolute IR and ER strength deficit percentage turned out similar between men and women (relative: $2 \%, p=$ $0.84, \eta \mathrm{p}^{2}=0.005$, no effect; absolute: $7 \%, p=0.395$, $\eta p^{2}=0.092$, medium effect).

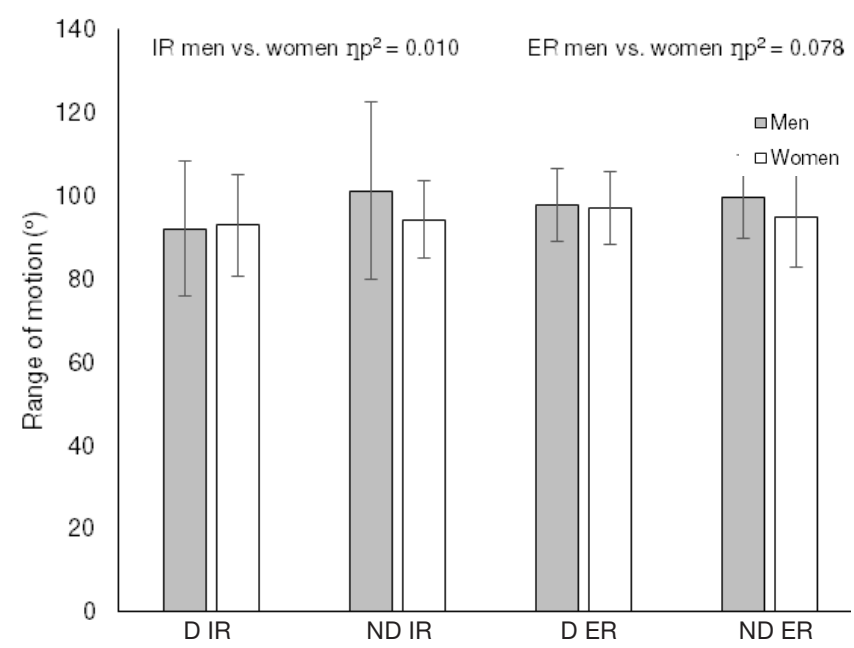

Figure 1. Shoulder internal (IR) and external (ER) rotation range of motion $\left(^{\circ}\right)$ in dominant and non-dominant shoulders for men and women volleyball players 


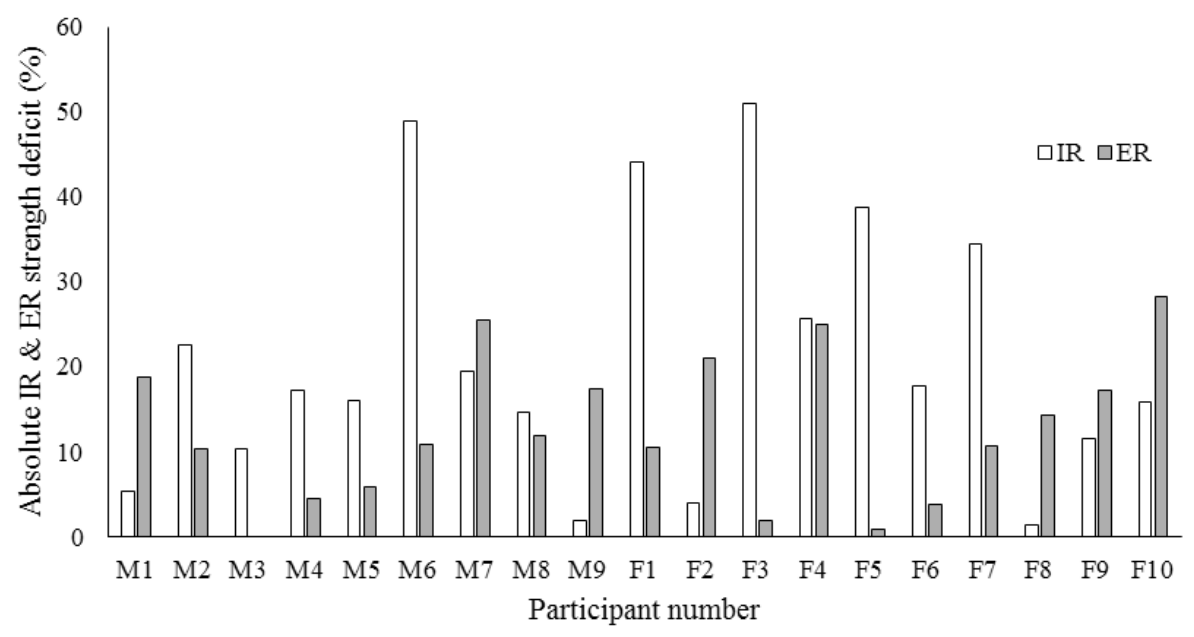

Figure 2. Shoulder internal (IR) and external (ER) rotation absolute strength deficit (\%) between dominant and non-dominant shoulders in male $(\mathrm{M})$ and female $(\mathrm{F})$ volleyball players

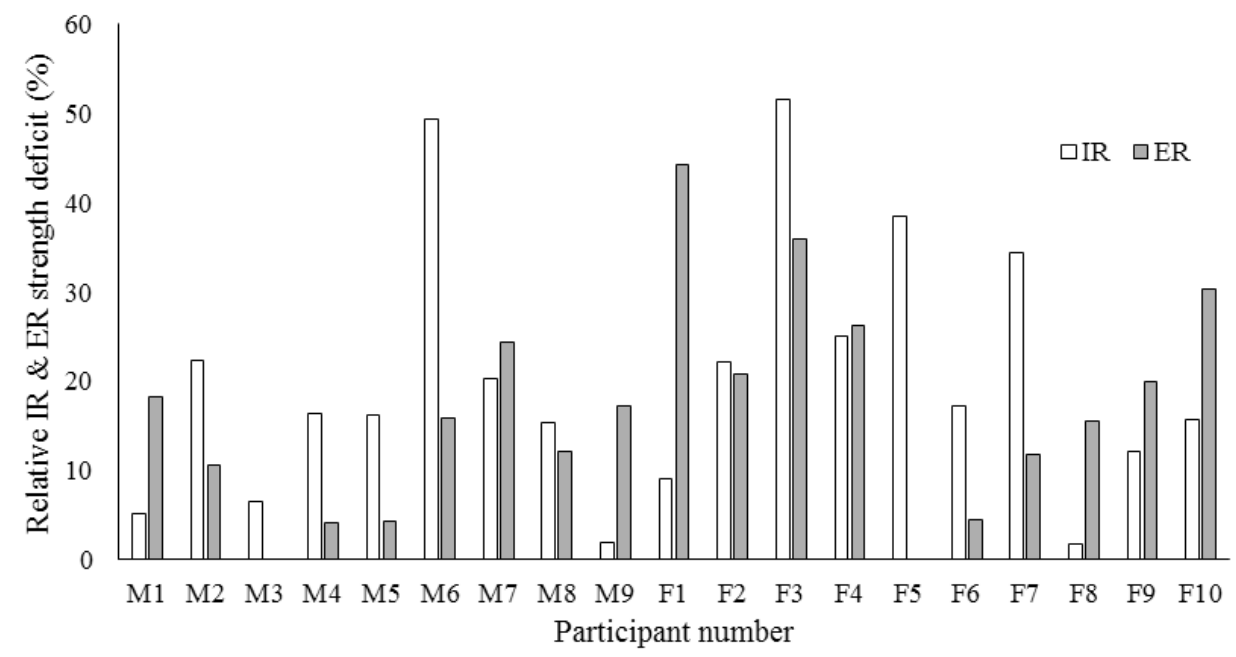

Figure 3. Shoulder internal (IR) and external (ER) rotation relative strength deficit (\%) between dominant and non-dominant shoulders in male $(\mathrm{M})$ and female $(\mathrm{F})$ volleyball players

Table 1. Internal (IR) and external (ER) rotators absolute and relative peak torque, ER/IR strength ratios, and strength deficit for dominant (D) and non-dominant (ND) shoulders in men and women volleyball players

\begin{tabular}{|c|c|c|c|c|c|c|c|c|c|c|c|}
\hline \multicolumn{2}{|c|}{ Men } & \multicolumn{2}{|c|}{ Women } & \multicolumn{3}{|c|}{ Dominance } & \multicolumn{3}{|c|}{ Sex } & \multicolumn{2}{|c|}{$\begin{array}{c}\text { Dominance } \\
\times \text { sex }\end{array}$} \\
\hline $\mathrm{D}$ & ND & $\mathrm{D}$ & ND & $\begin{array}{c}\% \\
\text { difference }\end{array}$ & $p$ & $\eta p^{2}$ & $\begin{array}{c}\% \\
\text { difference }\end{array}$ & $p$ & $\Pi p^{2}$ & $p$ & $\eta p^{2}$ \\
\hline $51.7 \pm 14.0^{* *}$ & $44.5 \pm 13.3^{* ¥}$ & $31.5 \pm 3.6^{* \Psi}$ & $24.2 \pm 6.9^{* \Psi}$ & 19 & 0.001 & 0.763 & 42 & 0.002 & 0.732 & 0.938 & 0.001 \\
\hline $32.7 \pm 9.8^{* Y}$ & $29.8 \pm 8.6^{* ¥}$ & $16.6 \pm 3.8^{* ¥}$ & $15.5 \pm 3.8^{* ¥}$ & 7 & 0.014 & 0.548 & 49 & 0.006 & 0.637 & 0.230 & 0.175 \\
\hline $0.65 \pm 0.17^{* Y}$ & $0.54 \pm 0.17^{* Y}$ & $0.48 \pm 0.09 * Y$ & $0.37 \pm 0.11^{* Y}$ & 20 & 0.001 & 0.771 & 29 & 0.026 & 0.480 & 0.924 & 0.001 \\
\hline $0.39 \pm 0.10^{¥}$ & $0.37 \pm 0.10^{¥}$ & $0.25 \pm 0.06^{¥}$ & $0.26 \pm 0.07^{¥}$ & 3 & 0.473 & 0.066 & 35 & 0.033 & 0.454 & 0.649 & 0.027 \\
\hline $0.64 \pm 0.12^{*}$ & $0.68 \pm 0.16^{*}$ & $0.53 \pm 0.11^{*}$ & $0.69 \pm 0.18^{*}$ & 17 & 0.012 & 0.570 & 7 & 0.395 & 0.092 & 0.189 & 0.205 \\
\hline $0.60 \pm 0.10^{*}$ & $0.71 \pm 0.15^{*}$ & $0.52 \pm 0.11^{*}$ & $0.76 \pm 0.35^{*}$ & 23 & 0.024 & 0.489 & 2 & 0.840 & 0.005 & 0.420 & 0.083 \\
\hline \multicolumn{2}{|c|}{$17.4 \pm 13.6$} & \multicolumn{2}{|c|}{$26.3 \pm 18.3$} & & & & 29 & \multicolumn{3}{|c|}{$0.195 d=0.46$} & \\
\hline \multicolumn{2}{|c|}{$11.7 \pm 7.9$} & \multicolumn{2}{|c|}{$13.4 \pm 9.5$} & & & & 12 & \multicolumn{3}{|c|}{$0.240 d=0.19$} & \\
\hline \multicolumn{2}{|c|}{$17.1 \pm 14.0$} & \multicolumn{2}{|c|}{$26.3 \pm 15.6$} & & & & 35 & \multicolumn{3}{|c|}{$0.414 d=0.61$} & \\
\hline \multicolumn{2}{|c|}{$11.9 \pm 14.0$} & \multicolumn{2}{|c|}{$17.4 \pm 11.5$} & & & & 32 & 0.240 & $d=0.55$ & & \\
\hline
\end{tabular}

* significant difference $(p<0.05)$ between D and ND shoulders

${ }^{¥}$ significant difference $(p<0.05)$ between men and women 
There were large between-subject differences in absolute and relative strength deficits (\%) between D and ND shoulders for both men and women volleyball players (Figures 2 and 3). Six participants displayed over $30 \%$ relative strength deficits between $\mathrm{D}$ and ND shoulders for either IR and ER. One participant presented absolute and relative strength deficits in both IR and ER between D and ND of less than 15\%.

\section{Discussion}

The aim of the study was to investigate the presence of asymmetries in the ROM and strength in the D and ND shoulders among university level men and women volleyball players. The main findings were that IR and ER strength was asymmetrical, in both men and women, in favour of the D side. Both men and women displayed asymmetrical external/internal ratios, with greater ratios present in the ND side. Furthermore, men and women exhibited similar levels of IR and ER strength deficits between D and ND shoulders.

Relative and absolute IR peak torque was found to be significantly different between D and ND (relative: $20 \%, p=0.001, \Pi p^{2}=0.771$, large effect; absolute: $19 \%, p=0.001, \eta p^{2}=0.763$, large effect), as well as between men and women (relative: $29 \%, p=0.026$, $\eta \mathrm{p}^{2}=0.480$, large effect; absolute: $42 \%, p=0.002$, $\eta p^{2}=0.732$, large effect). Our results agree with previous literature conducted in elite and high level volleyball in that the IR strength of the D shoulder is higher than that of the ND shoulder in men [17, 23, 25] and women volleyball players [17, 24]. Our results suggest that participating in volleyball for $7.3 \pm 3.2$ years and playing at a university standard can lead to the development of significant strength asymmetries between $D$ and ND shoulders in the absolute IR peak torque. When compared with the literature [17], the absolute and relative IR peak torque was found to be lower in our university level cohort than the higher level athletes. These lower values could be attributed to the difference in the number of hours they spent training a week. The participants in Hadzic et al.'s [17] study from the Slovenian first and second national divisions trained on average $1.7 \pm 0.6$ hours a day for the men and 1.3 \pm 0.6 hours a day for the women, where the male and female university level participants in the current study only spent $6.2 \pm 2.1$ hours a week. However, regardless of the training load, both men and women at university and higher level have significant asymmetries between $\mathrm{D}$ and ND shoulder IR peak torque.

When examining the relative and absolute ER peak torque, the current study proved significant differ- ences between D and ND (relative: $35 \%, p=0.033$, $\eta p^{2}=0.454$, large effect; absolute: $7 \%, p=0.014, \eta p^{2}=$ 0.548 , large effect), and men and women in the absolute peak torque $\left(49 \%, p=0.006, \eta p^{2}=0.637\right.$, large effect). The university level volleyball players presented similar asymmetries between D and ND as elite level athletes in studies by Wang et al. [25], Wang and Cochrane [23], and Michael et al. [26], but with the current results involving increased peak torque in the D shoulder when compared with ND. However, no difference was observed in relative ER peak torque between $\mathrm{D}$ and ND shoulders $\left(3 \%, p=0.473, \eta^{2}=\right.$ 0.066 , medium effect). The lack of any D-ND shoulder differences in relative peak ER torque potentially suggests that concentric IR, rather than ER, is the shoulder movement more likely to cause injury due to asymmetries in shoulder dominance. Further supporting this argument, in both the absolute and relative strength deficit the IR was $17.1-26.3 \%$, whereas the absolute and relative ER was $11.7-17.4 \%$. When compared with normal levels of strength asymmetry proposed by Reinold and Gill [20], the 17.1-26.3\% range of the IR is greater than the $9 \%$ threshold for increased risk of injury, whereas the ER strength deficit of $11.7-17.4 \%$ in favour of the D shoulder had a generally normal strength asymmetry (i.e. strength difference within $14 \%$ in favour of the ND shoulder [20]). Furthermore, both men and women displayed absolute and relative IR strength deficit greater than the suggested $15 \%$ inter-limb asymmetry threshold that can lead to an increased incidence of injury in the lower limbs [9-11]. However, 5 individuals in the current study displayed differences of more than $30 \%$, providing evidence that thresholds should be used with caution when assessing a group response. Therefore, both men and women university level volleyball players are potentially at risk of IR shoulder injury and isokinetic screening measures could be used to assess risk.

Unlike previous studies by Hadzic et al. [17] and Michael et al. [26], our results do not suggest significant important sex-related differences with regard to the ER strength asymmetry or any other variables measured. The absolute and relative ER/IR strength ratios were similar between sexes. With the majority of ratios sitting between the general recommendations (0.60-0.75) proposed by Ellenbecker and Davies [14], only the women D strength ratios were observed as slightly below (0.52-0.53). These ratios on the lower side of safe support previous literature [14, 17] in volleyball that highlights the ER weakness of the D (and also ND) shoulder in elite level, and now university level volleyball. One explanation for not observing dif- 
ferences between sexes could point at the large between-subject variation and reduced sample size. In men alone, there was a range of $1.9-49.0 \%$ between $\mathrm{D}$ and ND shoulders for absolute IR strength deficits. Such observations mirror recent recommendations by Bishop et al. [12] for an individual approach to data analysis when investigating inter-limb asymmetries. However, the differences observed between D and ND in both absolute and relative strength ratios, regardless of sex, support the recommendation that additional resistance training for concentric ER strength of the D and ND shoulder must be emphasised for university level volleyball players to address shoulder imbalances and asymmetries.

A study by Wang et al. [25] suggested a deficit in IR ROM in elite volleyball athletes and its strong association with shoulder pain. The current study implies that the IR and ER ROM was similar between D and ND, and between men and women. These findings support a study that reported similar ROM in D and ND shoulders for elite volleyball players [34]. Schwab and Blanch [34] proposed that a reason for no ROM differences in volleyball, when compared with other overhead activities, might be due to there being no extra weight of the ball/racket in the movement that could force the shoulder into more external rotation and potentially increase its ROM. As a consequence, ER and IR ROM may not be susceptible to asymmetrical injury risk in university level volleyball players.

The main practical application of the current study is that it is recommended that additional resistance training for concentric ER strength of the D and ND shoulder should be emphasised for university level volleyball players to address shoulder strength imbalances and asymmetries. Furthermore, the large between-subject response highlights the potential need for increased analysis at the individual level and the necessity to use generalised injury thresholds with caution. Future studies should focus on determining if detrimental effects are shown in a variety of tasks associated with shoulder movement in volleyball to examine if thresholds exist that are related to performance or injury. Moreover, there is a need for longitudinal studies to investigate how the asymmetries of volleyball players change during a season or training intervention.

The first limitation of the current study was the sample size, which made it not possible to comment on the effect that playing position might have on strength and ROM asymmetries. Furthermore, the large between-subject variance in results had the potential to mask group-based statistics. Secondly, the study lacked the examination of eccentric contractions during internal and external shoulder rotations, which might provide further insight into potential injury risk factors. Thirdly, the use of a self-directed warm-up could have interfered with the results, as some participants could have used a more effective warm-up routine than others. Finally, an intra-observer test was not conducted for the shoulder internal or external ROM. An intra-observer test would have enabled reliability results to be presented for these measures.

\section{Conclusions}

In conclusion, the results of our study highlight that university level men and women volleyball players exhibit similar levels of significant asymmetry in IR and ER strength between D and ND spiking arms. As a consequence of the observed shoulder asymmetries, both sexes at this level of competition are potentially at risk of developing injuries related to IR of the shoulder. It is recommended that additional resistance training for concentric ER strength of the D and ND shoulder should be emphasised for university level volleyball players to address shoulder strength imbalances and asymmetries. Furthermore, isokinetic shoulder screening could be used to reveal information about possible risk factors for shoulder injuries.

\section{Disclosure statement}

No author has any financial interest or received any financial benefit from this research.

\section{Conflict of interest}

The authors state no conflict of interest.

\section{References}

1. Keeley DW, Plummer HA, Oliver GD. Predicting asymmetrical lower extremity strength deficits in collegeaged men and women using common horizontal and vertical power field tests: a possible screening mechanism. J Strength Cond Res. 2011;25(6):1632-1637; doi: 10.1519/JSC.0b013e3181ddf690.

2. Bishop C, Turner A, Read P. Effects of inter-limb asymmetries on physical and sports performance: a systematic review. J Sports Sci. 2018;36(10):1135-1144; doi: 10.1080/02640414.2017.1361894.

3. Challoumas D, Stavrou A, Dimitrakakis G. The volleyball athlete's shoulder: biomechanical adaptations and injury associations. Sports Biomech. 2017;16(2):220 237; doi: 10.1080/14763141.2016.1222629.

4. Lo YP, Hsu YC, Chan KM. Epidemiology of shoulder impingement in upper arm sports events. Br J Sports Med. 1990;24(3);173-177; doi: 10.1136/bjsm.24.3.173. 
5. Seminati E, Minetti AE. Overuse in volleyball training/ practice: a review on shoulder and spine-related injuries. Eur J Sport Sci. 2013;13(6):732-743; doi: 10.1080/ 17461391.2013.773090.

6. Kyritsis P, Bahr R, Landreau P, Miladi R, Witvrouw E. Likelihood of ACL graft rupture: not meeting six clinical discharge criteria before return to sport is associated with a four times greater risk of rupture. Br J Sports Med. 2016;50(15):946-951; doi: 10.1136/bjsports-2015-095908.

7. Rohman E, Steubs JT, Tompkins M. Changes in involved and uninvolved limb function during rehabilitation after anterior cruciate ligament reconstruction: implications for Limb Symmetry Index measures. Am J Sports Med. 2015;43(6):1391-1398; doi: 10.1177/ 0363546515576127.

8. Dos Santos KB, Bento PCB, Pereira G, Payton C, Rodacki ALF. Front crawl swimming performance and bilateral force asymmetry during land-based and tethered swimming tests. J Sports Sci Med. 2017;16(4):574-580.

9. Barber SD, Noyes FR, Mangine RE, McCloskey JW, Hartman W. Quantitative assessment of functional limitations in normal and anterior cruciate ligamentdeficient knees. Clin Orthop Relat Res. 1990;255:204214; doi: 10.1097/00003086-199006000-00028.

10. Grindem H, Logerstedt D, Eitzen I, Moksnes H, Axe MJ, Snyder-Mackler L, et al. Single-legged hop tests as predictors of self-reported knee function in nonoperatively treated individuals with anterior cruciate ligament injury. Am J Sports Med. 2011;39(11):2347-2354; doi: 10.1177/0363546511417085.

11. Impellizzeri FM, Rampinini E, Maffiuletti N, Marcora SM. A vertical jump force test for assessing bilateral strength asymmetry in athletes. Med Sci Sports Exerc. 2007;39(11):2044-2050; doi: 10.1249/mss.0b013e31 $814 \mathrm{fb} 55 \mathrm{c}$.

12. Bishop C, Lake J, Loturco I, Papadopoulos K, Turner A, Read P. Interlimb asymmetries: the need for an individual approach to data analysis. J Strength Cond Res. 2018; doi: 10.1519/JSC.0000000000002729.

13. Bere T, Kruczynski J, Veintimilla N, Hamu Y, Bahr R. Injury risk is low among world-class volleyball players: 4-year data from the FIVB Injury Surveillance System. Br J Sports Med. 2015;49(17):1132-1137; doi: 10.1136/ bjsports-2015-094959.

14. Ellenbecker TS, Davies GJ. The application of isokinetics in testing and rehabilitation of the shoulder complex. J Athl Train. 2000;35(3):338-350; doi: 10.1016/ B978-044306701-3.50057-8.

15. Hamill J, Knutzen KM. Biomechanical basis of human movement, $2^{\text {nd }}$ ed. Philadelphia: Lippincott Williams \& Wilkins; 2003.

16. Noffal GJ. Isokinetic eccentric-to-concentric strength ratios of the shoulder rotator muscles in throwers and nonthrowers. Am J Sports Med. 2003;31(4):537-541; doi: 10.1177/03635465030310041001.
17. Hadzic V, Sattler T, Veselko M, Markovic G, Dervisevic E. Strength asymmetry of the shoulders in elite volleyball players. J Athl Train. 2014;49(3):338-344; doi: 10.4085/1062-6050-49.2.05.

18. Andrade Mdos S, Fleury AM, de Lira CA, Dubas JP, da Silva AC. Profile of isokinetic eccentric-to-concentric strength ratios of shoulder rotator muscles in elite female team handball players. J Sports Sci. 2010;28(7): 743-749; doi: 10.1080/02640411003645687.

19. Stickley CD, Hetzler RK, Freemyer BG, Kimura IF. Isokinetic peak torque ratios and shoulder injury history in adolescent female volleyball athletes. J Athl Train. 2008;43(6):571-577; doi: 10.4085/1062-605043.6.571.

20. Reinold MM, Gill TJ. Current concepts in the evaluation and treatment of the shoulder in overhead-throwing athletes, part 1: physical characteristics and clinical examination. Sports Health. 2010;2(1):39-50; doi: 10.1177/1941738109338548.

21. Witvrouw E, Cools A, Lysens R, Cambier D, Vanderstraeten G, Victor J, et al. Suprascapular neuropathy in volleyball players. Br J Sports Med. 2000;34(3):174180; doi: 10.1136/bjsm.34.3.174.

22. Gozlan G, Bensoussan L, Coudreuse JM, Fondarai J, Gremeaux V, Viton JM, et al. Isokinetic dynamometer measurement of shoulder rotational strength in healthy elite athletes (swimming, volley-ball, tennis): comparison between dominant and nondominant shoulder [in French]. Ann Readapt Med Phys. 2006;49(1):8-15; doi: 10.1016/j.annrmp.2005.07.001.

23. Wang HK, Cochrane T. Mobility impairment, muscle imbalance, muscle weakness, scapular asymmetry and shoulder injury in elite volleyball athletes. J Sports Med Phys Fitness. 2001;41(3):403-410.

24. Alfredson H, Pietilä T, Lorentzon R. Concentric and eccentric shoulder and elbow muscle strength in female volleyball players and non-active females. Scand J Med SciSports.1998;8(5):265-270; doi:10.1111/j.1600-0838. 1998.tb00481.x.

25. Wang H, Macfarlane A, Cochrane T. Isokinetic performance and shoulder mobility in elite volleyball athletes from the United Kingdom. Br J Sports Med. 2000;34(1): 39-43; doi: 10.1136/bjsm.34.1.39.

26. Michael J, König D, Hessling U, Popken F, Eysel P. Results of shoulder isokinetic testing in volleyball players [in German]. Sportverletz Sportschaden. 2003;17(2): 71-74; doi: 10.1055/s-2003-40133.

27. Borsa PA, Laudner KG, Sauers EL. Mobility and stability adaptations in the shoulder of the overhead athlete: a theoretical and evidence-based perspective. SportsMed.2008;38(1):17-36, doi: 10.2165/00007256200838010-00003.

28. Myers JB, Laudner KG, Pasquale MR, Bradley JP, Lephart SM. Scapular position and orientation in throwing athletes. Am J Sports Med. 2005;33(2):263-271; doi: 10.1177/0363546504268138. 
29. Yildiz Y, Aydin T, Sekir U, Kiralp MZ, Hazneci B, Kalyon TA. Shoulder terminal range eccentric antagonist/ concentric agonist strength ratios in overhead athletes. Scand J Med Sci Sports. 2005;16(3):174-180; doi: 10.1111/j.1600-0838.2005.00471.x.

30. Friery KB, Bishop P. Long-term impact of athletic participation on physical capabilities. J Exerc Physiol Online. 2007;10(1):34-46.

31. Simon JE, Docherty CL. The impact of previous athletic experience on current physical fitness in former collegiate athletes and noncollegiate athletes. Sports Health. 2017;9(5):462-468; doi: 10.1177/194173811 7705311.

32. Kolber MJ, Hanney WJ. The reliability and concurrent validity of shoulder mobility measurements using a digital inclinometer and goniometer: a technical report. Int J Sports Phys Ther. 2012;7(3):306-313.

33. Richardson JTE. Eta squared and partial eta squared as measures of effect size in educational research. Educ Res Rev. 2011;6(2):135-147; doi: 10.1016/j.edurev.2010. 12.001 .

34. Schwab LM, Blanch P. Humeral torsion and passive shoulder range in elite volleyball players. Phys Ther Sport. 2009;10(2):51-56; doi: 10.1016/j.ptsp.2008.11.006. 